Bull. Austral. Math. Soc.

$70 \mathrm{H} 20,46 \mathrm{E} 35,35 \mathrm{D} 10,41 \mathrm{~A} 25$

VoL. 51 (1995) [195-213]

\title{
REGULARITY FOR HAMILTON-JACOBI EQUATIONS VIA APPROXIMATION
}

\author{
Bum IL Hong
}

\begin{abstract}
We prove new regularity results for solutions of first-order partial differential equations of Hamilton-Jacobi type posed as initial value problems on the real line. We show that certain spaces determined by quasinorms related to the solution's approximation properties in $C(\mathbb{R})$ by continuous, piecewise quadratic polynomial functions are invariant under the action of the differential equation. As a result, we show that solutions of Hamilton-Jacobi equations have enough regularity to be approximated well in $C(\mathbf{R})$ by moving-grid finite element methods. The preceding results depend on a new stability theorem for Hamilton-Jacobi equations in any number of spatial dimensions.
\end{abstract}

\section{INTRODUCTION}

Analysis by the method of characteristics shows that if $f$ and $u_{0}$ are smooth and $u_{0}$ has compact support, then the Hamilton-Jacobi equation

$$
u_{t}+f(\nabla u)=0 \quad x \in \mathbb{R}^{N}, t>0
$$

$$
u(x, 0)=u_{0}(x), \quad x \in \mathbb{R}^{N}
$$

has a unique $C^{1}$ solution $u$ on some maximal time interval $0 \leqslant t<T$ for which $\lim _{t \rightarrow T} u(x, t)$ exists uniformly; but this limiting function is not continuously differentiable. Thus, $u_{x}$ becomes discontinuous at $t=T$, and it is therefore necessary to deal with nonsmooth solutions if we want a solution of (H-J) that is defined for all $t>0$. If the equation is understood in the "almost-everywhere" sense, one can produce Lipschitz continuous functions on $\mathbb{R}^{N}$ which satisfy (H-J) for all positive time. Though "generalised" solutions in this sense are not unique, both existence and uniqueness of generalised solutions that satisfy a so-called "viscosity" condition were shown by Crandall and Lions [3]. The viscosity solution of (H-J) is the limit as $\epsilon$ tends to

\section{Received 6 April 1994}

This paper was written after extensive conversation with B. Lucier and J. Douglas, Jr. at Purdue University. I am deeply indebted to them. I also thank R. DeVore at the University of South Carolina for his nice suggestions.

\footnotetext{
Copyright Clearance Centre, Inc. Serial-fee code: 0004-9729/95 \$A2.00+0.00.
} 
zero of the solution of (H-J) with the right-hand side replaced by $\epsilon \Delta u$ (hence the name "viscosity"). Crandall, Evans, and Lions [2] provide a perhaps simpler introduction to the subject, and the book by Lions [11] gives theory and references. Souganidis [15] also discusses the existence of a unique viscosity solution under certain assumptions. Crandall and Lions [4] establish the convergence of a general class of finite difference schemes to the viscosity solution of (H-J) and give explicit error estimates. Souganidis [14] establishes several results concerning the convergence of explicit and implicit finite difference schemes (with error estimates) under certain assumptions. Jensen and Souganidis [8] consider regularity for Hamilton-Jacobi equations in one space dimension by studying the structure of the singularities of solutions.

In one space dimension, the equation (H-J) is closely related to hyperbolic conservation laws. This relation is very simple: if $u$ solves $(H-J)$, then $v=u_{x}$ solves a scalar conservation law

(C)

$$
v_{t}+f(v)_{x}=0, \quad x \in \mathbb{R}, t>0
$$

$$
v(x, 0)=v_{0}(x)=u_{0}^{\prime}(x), \quad x \in \mathbb{R}
$$

This can be proved by noting that the entropy solution of $(C)$ is also the limit as $\epsilon$ tends to zero of the solution of (C) with the right hand side replaced by $\epsilon v_{x x}$; see Kružhkov $[\mathbf{9}]$.

\section{VISCOSITY SOLUTIONS OF (H-J)}

To repeat, one cannot in general find a classical solution of (H-J) on $\mathbb{R} \times[0, \infty)$, while Lipschitz continuous "generalised solutions" in the almost-everywhere sense exist but are not unique. If $u$ and $v$ are generalised solutions of (H-J), then so are $\min (u, v)$ and $\max (u, v)$. In fact, if the problem is nonlinear, one can expect infinitely many generalised solutions. The uniqueness problem is resolved by introducing a notion of viscosity. Crandall and Lions [3] give the notion of viscosity and uniqueness results for viscosity solutions.

DEFINITION 2.1: A viscosity subsolution (respectively, supersolution) of (H-J) with $f \in C\left(\mathbb{R}^{N}\right)$ is a function $u \in C\left(\mathbb{R}^{N} \times[0, \infty)\right)$ such that for every $\phi \in$ $C^{1}\left(\mathbb{R}^{N} \times(0, \infty)\right)$ and $T>0$ :

$$
\begin{aligned}
& \text { If }\left(x_{0}, t_{0}\right) \text { is a local maximum point of } u-\phi \\
& \text { on } \mathbb{R}^{N} \times(0, T] \text {, then }
\end{aligned}
$$$$
\phi_{t}\left(x_{0}, t_{0}\right)+f\left(\nabla \phi\left(x_{0}, t_{0}\right)\right) \leqslant 0 .
$$ 
( respectively,

If $\left(x_{0}, t_{0}\right)$ is a local maximum point of $u-\phi$ on $\mathbb{R}^{N} \times(0, T)$, then

$$
\left.\phi_{t}\left(x_{0}, t_{0}\right)+f\left(\nabla \phi\left(x_{0}, t_{0}\right)\right) \geqslant 0 .\right)
$$

Definition 2.2: A viscosity solution of (H-J) is a function $u \in C\left(\mathbb{R}^{N} \times[0, \infty)\right)$ for which both (2.1.1) and (2.1.2) hold (that is, $u$ is both a viscosity subsolution and a viscosity supersolution).

REMARK. If $u$ is a classical solution of (H-J), then it is a viscosity solution, and if $u$ is a viscosity solution of (H-J), then $u_{t}\left(x_{0}, t_{0}\right)+f\left(\nabla u\left(x_{0}, t_{0}\right)\right)=0$ at any point $\left(x_{0}, t_{0}\right)$ where $u$ is differentiable.

LEMma 2.3. Suppose that $u(x, t)$ and $v(x, t)$ are viscosity solutions of

$$
\begin{array}{ll}
w_{t}+f(\nabla w)=0, & x \in \mathbb{R}^{N}, t>0, \\
w(x, 0)=w_{0}(x), & x \in \mathbb{R}^{N},
\end{array}
$$

with initial data $u_{0}(x)$ and $v_{0}(x)$ respectively. Then

$$
\begin{gathered}
\|u(\cdot, t)-v(\cdot, t)\|_{L^{\infty}\left(\mathbb{R}^{N}\right)} \leqslant\left\|u_{0}-v_{0}\right\|_{L^{\infty}\left(\mathbb{B}^{N}\right)} \quad \text { and } \\
\inf _{\mathbb{R}^{N}} u_{0}(x) \leqslant t f(0)+u(\cdot, t) \leqslant \sup _{\mathbb{R}^{N}} u_{0}(x) .
\end{gathered}
$$

Proof: See [2] and [4].

Lemma 2.4. The function $v$ is the viscosity solution of $v_{t}+g(\nabla v)=0$ if and only if $u=-v$ is the viscosity solution of $u_{t}-g(-\nabla u)=0$.

Proof: $(\Rightarrow)$ (1) Suppose that $u=-v$. Let $\phi \in C^{1}\left(\mathbb{R}^{N} \times(0, \infty)\right)$ and let $v-\phi$ attain a local maximum at $\left(x_{0}, t_{0}\right)$. Then

$$
\phi_{t}\left(x_{0}, t_{0}\right)+g\left(\nabla \phi\left(x_{0}, t_{0}\right)\right) \leqslant 0
$$

Since $-(v-\phi)=-v-(-\phi)=-(-\phi)$ and $u-(-\phi)$ attains a local minimum at $\left(x_{0}, t_{0}\right)$, by $(\mathrm{A})$,

$$
-\phi_{t}\left(x_{0}, t_{0}\right)-g\left(-\left(-\nabla \phi\left(x_{0}, t_{0}\right)\right)\right)=-\phi_{t}\left(x_{0}, t_{0}\right)-g\left(\nabla \phi\left(x_{0}, t_{0}\right)\right) \geqslant 0 .
$$

Therefore, $u=-v$ is a viscosity supersolution of $u_{t}-g(-\Delta u)=0$. 
(2) Let $v-\phi$ attain a local minimum at $\left(x_{0}, t_{0}\right)$. Then

$$
\phi_{t}\left(x_{0}, t_{0}\right)+g\left(\nabla \phi\left(x_{0}, t_{0}\right)\right) \geqslant 0 .
$$

Since $-(v-\phi)=-v-(-\phi)=u-(-\phi)$ and $u-(-\phi)$ attains a local maximum at $\left(x_{0}, t_{0}\right)$, by $(\mathrm{B})$,

$$
-\phi_{t}\left(x_{0}, t_{0}\right)-g\left(-\left(-\nabla \phi\left(x_{0}, t_{0}\right)\right)\right)=-\phi_{t}\left(x_{0}, t_{0}\right)-g\left(\nabla \phi\left(x_{0}, t_{0}\right)\right) \leqslant 0 .
$$

Therefore, $u=-v$ is a viscosity subsolution of $u_{t}-g(-\nabla u)=0$. By (1) and (2), the conclusion follows.

$(\Leftarrow)$ By the same argument, the other part follows.

\section{STABILITY OF TWO VISCOSITY SOLUTIONS}

We prove that viscosity solutions are stable under changes in the nonlinear flux functions as well as changes in the initial data. The following result is similar to the stability result of Lucier [12] for conservation Laws. His result holds only for one space dimension, but mine holds for several space dimensions.

THEOREM 3.1. Let $u$ and $v$ be the viscosity solutions of

$$
\begin{array}{ll}
u_{t}+f(\nabla u)=0, & x \in \mathbb{R}^{N}, t>0 \\
u(x, 0)=u_{0}(x), & x \in \mathbb{R}^{N},
\end{array}
$$

and

$$
\begin{array}{ll}
v_{t}+g(\nabla v)=0, & x \in \mathbb{R}^{N}, t>0 \\
v(x, 0)=v_{0}(x), & x \in \mathbb{R}^{N},
\end{array}
$$

respectively, where $f$ and $g$ are Lipschitz continuous, $f(0)=g(0)=0$, and $u_{0}$ and $v_{0}$ are bounded and Lipschitz continuous. Then for any $t>0$,

$$
\|u(\cdot, t)-v(\cdot, t)\|_{L^{\infty}\left(\mathbb{R}^{N}\right)} \leqslant\left\|u_{0}-v_{0}\right\|_{L^{\infty}\left(\mathbb{\Phi}^{N}\right)}+t\|f-g\|_{L^{\infty}\left(\mathbb{R}^{N}\right)} .
$$

Theorem 3.1 is the main result of this section. To prove that, we prepare with two lemmas.

LEMMA 3.2. Let $u$ and $w$ be the viscosity solutions of

$$
\begin{array}{ll}
u_{t}+f(\nabla u)=0, & x \in \mathbb{R}^{N}, t>0, \\
u(x, 0)=u_{0}(x), & x \in \mathbb{R}^{N},
\end{array}
$$


and

$$
\begin{array}{ll}
w_{t}+g(\nabla w)=0, & x \in \mathbb{R}^{N}, t>0 \\
w(x, 0)=u_{0}(x), & x \in \mathbb{R}^{N},
\end{array}
$$

respectively, where $f$ and $g$ are Lipschitz continuous, $f(0)=g(0)=0$, and $u_{0}$ is bounded and Lipschitz continuous. Then for all positive time $t$,

$$
\|u(\cdot, t)-w(\cdot, t)\|_{L^{\infty}\left(\mathbb{R}^{N}\right)} \leqslant t\|f-g\|_{L^{\infty}\left(\mathbb{R}^{N}\right)} \cdot
$$

Theorem 3.1 follows by combining Lemma 2.3 and Lemma 3.2 and Lemma 3.2 follows from Lemma 3.3.

LEMMA 3.3. Assume that $u$ and $w$ are as in Lemma 3.2. Let $M=\left\|u_{0}\right\|_{L^{\infty}\left(\mathbb{R}^{N}\right)}$ and let $\eta(z)$ be a smooth function on $\mathbb{R}$ such that $\eta(-z)=\eta(z), 0 \leqslant \eta(z) \leqslant 1, \eta(0)=1$ and $\eta(z)=0$ if $|z|>1$. Suppose that

$$
\sigma:=\sup _{\mathbb{R}^{N} \times[0, T]}(u(x, t)-w(x, t))>0 .
$$

For any $\lambda>2$ and $\epsilon>0$ define

$$
\psi(x, t, y, s)=u(x, t)-w(y, s)-\frac{\sigma}{\lambda T}(t+s)+\left(5 M+\frac{\sigma}{2}\right) \beta_{\epsilon}(x-y, t-x),
$$

where $\beta_{\epsilon}(x, t)$ is defined on $\mathbb{R}^{N} \times \mathbb{R}$ by $\beta_{\epsilon}(x, t)=\eta(t / \epsilon) \prod_{i=1}^{N} \eta\left(x_{i} / \epsilon\right)$. If

$$
\sup _{\substack{|x| \geqslant r \\ 0 \leqslant t \leqslant T}}|u(x, t)| \text { and } \sup _{\substack{|x| \geqslant r \\ 0 \leqslant t \leqslant T}}|w(x, t)| \rightarrow 0 \quad \text { as } r \rightarrow \infty
$$

then for any $\lambda>2$, there exists an $\epsilon>0$ and a point $\left(x_{0}, t_{0}, y_{0}, s_{0}\right) \in\left(\mathbb{R}^{N} \times(0, T]\right)^{2}$ such that $\psi\left(x_{0}, t_{0}, y_{0}, s_{0}\right) \geqslant \psi(x, t, y, s)$ on $\left(\mathbb{R}^{N} \times(0, T]\right)^{2}$.

Proof: Fix $\lambda>2$ and $\epsilon>0$. If there is a sequence $\left\{\left(x_{i}, t_{i}, y_{i}, s_{i}\right)\right\}_{i \geqslant 1}$ in $\left(\mathbb{R}^{N} \times[0, T]\right)^{2}$ such that

$$
\psi\left(x_{i}, t_{i}, y_{i}, s_{i}\right) \rightarrow \sup _{\left(\mathbb{R}^{N} \times[0, T]\right)^{2}} \psi
$$

then $\left(x_{i}, t_{i}, y_{i}, s_{i}\right)$ remains bounded by the following arguments. 
First,

$$
\begin{aligned}
\sup _{\left(\mathbb{R}^{N} \times[0, T]\right)^{2}} \psi & \geqslant u(x, t)-w(x, t)-\frac{\sigma}{\lambda T}(t+t)+\left(5 M+\frac{\sigma}{2}\right) \beta_{\epsilon}(x-x, t-t) \\
& =u(x, t)-w(x, t)-\frac{2 \sigma t}{\lambda T}+5 M+\frac{\sigma}{2} \quad \text { for all }(x, t) \in \mathbb{R}^{N} \times[0, T]
\end{aligned}
$$

Therefore

$$
\begin{aligned}
\sup _{\left(\mathbb{R}^{N} \times[0, T]\right)^{2}} \psi & \geqslant \sup _{\left(\mathbb{R}^{N} \times[0, T]\right)}(u(x, t)-w(x, t))-\frac{2 \sigma}{\lambda}+5 M+\frac{\sigma}{2} \\
& =\sigma-\frac{2 \sigma}{\lambda}+5 M+\frac{\sigma}{2} \\
& =5 M+\sigma\left(\frac{3}{2}-\frac{2}{\lambda}\right) .
\end{aligned}
$$

If $\beta_{\varepsilon}(x-y, t-s)=0$, then

$$
\begin{aligned}
\psi(x, t, y, s) & =u(x, t)-w(y, s)-\frac{\sigma}{\lambda T}(t+s) \\
& \leqslant u(x, t)-w(y, s) \\
& \leqslant 2 M
\end{aligned}
$$

Hence, (3.3.2) implies that $\beta_{\epsilon}\left(x_{i}-y_{i}, t_{i}-s_{i}\right)>0$ for large $i$, whence $\left|x_{i}-y_{i}\right|<\epsilon$ and $\left|t_{i}-s_{i}\right|<\epsilon$. If $\left|x_{i}\right| \rightarrow \infty$ and $\left|y_{i}\right| \rightarrow \infty$, then

$$
\lim _{i \rightarrow \infty} \sup _{\left(\mathbb{R}^{N} \times[0, T]\right)^{2}} \psi\left(x_{i}, t_{i}, y_{i}, s_{i}\right) \leqslant 5 M+\frac{\sigma}{2} \quad \text { by (3.3.1). }
$$

This contradicts (3.3.2) and (3.3.3). Therefore, $\left\{\left(x_{i}, t_{i}, y_{i}, x_{i}\right)\right\}_{i \geqslant 1}$ is a bounded sequence and there is a convergent subsequence of $\left\{\left(x_{i}, t_{i}, y_{i}, s_{i}\right)\right\}_{i \geqslant 1}$. Let $\left(x_{0}, t_{0}, y_{0}, s_{0}\right)$ be the limit of the above subsequence. We shall show that we can pick $\epsilon$ such that $t_{0}>0$ and $s_{0}>0$.

We write

$$
\begin{aligned}
\psi\left(x_{0}, t_{0}, y_{0}, s_{0}\right)= & u\left(x_{0}, t_{0}\right)-u\left(x_{0}, 0\right)+u\left(x_{0}, 0\right)-w\left(x_{0}, 0\right) \\
& +w\left(x_{0}, 0\right)-w\left(y_{0}, 0\right)+w\left(y_{0}, 0\right)-w\left(y_{0}, s_{0}\right) \\
& -\frac{\sigma}{\lambda T}\left(t_{0}+s_{0}\right)+\left(5 M+\frac{\sigma}{2}\right) \beta_{\epsilon}\left(x_{0}-y_{0}, t_{0}-s_{0}\right)
\end{aligned}
$$

We have $\left|u\left(x_{0}, t_{0}\right)-u\left(x_{0}, 0\right)\right| \leqslant L_{f} t_{0}$ where $L_{f}:=\sup \left\{\left.|f(\rho)||| \rho|\leqslant| \nabla u_{0}\right|_{L^{\infty}\left(\mathbf{R}^{N}\right)}\right\}$ (and similarly $\left.\left|w\left(y_{0}, 0\right)-w\left(y_{0}, s_{0}\right)\right| \leqslant L_{g} s_{0}\right), u\left(x_{0}, 0\right)-w\left(x_{0}, 0\right)=0$, and 
$\left|w\left(x_{0}, 0\right)-w\left(y_{0}, 0\right)\right| \leqslant \omega\left(w_{0}, \epsilon\right)$, where $\omega\left(w_{0}, \cdot\right)$ is the modulus of smoothness of $w_{0}$. Therefore, if $\max \left(t_{0}, s_{0}\right)=\delta$, then

$$
\psi\left(x_{0}, t_{0}, y_{0}, s_{0}\right) \leqslant\left(L_{f}+L_{g}\right) \delta+\omega\left(w_{0}, \epsilon\right)+\left(5 M+\frac{\sigma}{2}\right) .
$$

From this inequality and (3.3.3) we see that

$$
5 M+\sigma\left(\frac{3}{2}-\frac{2}{\lambda}\right) \leqslant\left(L_{f}+L_{g}\right) \delta+\omega\left(w_{0}, \epsilon\right)+\left(5 M+\frac{\sigma}{2}\right)
$$

or

$$
\sigma\left(1-\frac{2}{\lambda}\right) \leqslant\left(L_{f}+L_{g}\right) \delta+\omega\left(w_{0}, \epsilon\right) .
$$

Assume that $\epsilon_{0}$ satisfies

$$
\omega\left(w_{0}, \epsilon_{0}\right) \leqslant \frac{\sigma}{2}\left(1-\frac{2}{\lambda}\right)
$$

then, for any $0<\epsilon \leqslant \epsilon_{0}$, we have

$$
\delta \geqslant \frac{1}{L_{f}+L_{g}} \frac{\sigma}{2}\left(1-\frac{2}{\lambda}\right)>0 .
$$

This is independent of $\epsilon$ for $0<\epsilon \leqslant \epsilon_{0}$; so choose $\epsilon<\delta / 2$. Then we have $\max \left(t_{0}, s_{0}\right)=$ $\delta$ and $\left|t_{0}-s_{0}\right| \leqslant \epsilon<\delta / 2$, so $\min \left(t_{0}, s_{0}\right)>\delta / 2>0$.

REMARK. If $u$ is the viscosity solution of (H-J) with $\left\|u_{0}\right\|_{L^{\infty}\left(\mathbb{B}^{N}\right)} \leqslant M$ for some $M>0$, then, by Lemma $2.3,\|u(\cdot, t)\|_{L^{\infty}\left(\mathbb{R}^{N}\right)} \leqslant M$.

Proof of Lemma 3.2: We shall prove that $\sigma$, defined in Lemma 3.3, satisfies

$$
\sigma \leqslant T\|F-g\|_{L^{\infty}\left(\mathbb{R}^{N}\right)} .
$$

By symmetry in $u$ and $w$, we see that this implies

$$
\|u-w\|_{L^{\infty}\left(\mathbb{R}^{N} \times[0, T]\right)} \leqslant T\|f-g\|_{L^{\infty}\left(\mathbb{R}^{N}\right)} .
$$

We first assume that

$$
\sup _{\substack{|x| \geqslant r \\ 0 \leqslant t \leqslant T}}|u(x, t)| \text { and } \sup _{\substack{|x| \geqslant r \\ 0 \leqslant t \leqslant T}}|w(x, t)| \rightarrow 0 \quad \text { as } r \rightarrow \infty .
$$

If $\sigma=0$, then we are done. Otherwise, by Lemma 3.3, for any $\lambda>2$ we can find an $\epsilon>0$ and a point $\left(x_{0}, t_{0}, y_{0}, s_{0}\right) \in\left(\mathbb{R}^{N} \times(0, T]\right)^{2}$ such that

$$
u(x, t)-\left(w\left(y_{0}, s_{0}\right)+\frac{\sigma}{\lambda T}\left(t+s_{0}\right)-\left(5 M+\frac{\sigma}{2}\right) \beta_{\epsilon}\left(x-y_{0}, t-s_{0}\right)\right)
$$


attains a local maximum at $\left(x_{0}, t_{0}\right)$, whence

$$
\begin{aligned}
\frac{\sigma}{\lambda T} & -\left(5 M+\frac{\sigma}{2}\right) \frac{\partial \beta_{\epsilon}}{\partial t}\left(x_{0}-y_{0}, t_{0}-s_{0}\right) \\
& +f\left(-\left(5 M+\frac{\sigma}{2}\right) \nabla_{x} \beta_{\varepsilon}\left(x_{0}-y_{0}, t_{0}-s_{0}\right)\right) \leqslant 0 .
\end{aligned}
$$

Similarly

$$
-w(y, s)-\left(-u\left(x_{0}, t_{0}\right)+\frac{\sigma}{\lambda T}\left(t_{0}+s\right)-\left(t M+\frac{\sigma}{2}\right) \beta_{\epsilon}\left(x_{0}-y, t_{0}-s\right)\right)
$$

attains a local maximum at $\left(y_{0}, s_{0}\right)$. Let $z(y, s)=-w(y, s)$ and let

$$
\phi(y, s)=u\left(x_{0}, t_{0}\right)-\frac{\sigma}{\lambda T}\left(t_{0}+s\right)+\left(5 M+\frac{\sigma}{2}\right) \beta_{\epsilon}\left(x_{0}-y, t_{0}-s\right) .
$$

Then $z(y, s)-(-\phi(y, s))$ attain a local maximum at $\left(y_{0}, s_{0}\right)$. Since Lemma 2.4 says that $z(y, s)$ is the visocsity solution of $z_{s}-g\left(-\nabla_{y} z\right)=0$ and $-\phi_{s}\left(y_{0}, s_{0}\right)-$ $g\left(\nabla_{y} \phi\left(y_{0}, s_{0}\right)\right) \leqslant 0$, we have

$$
\begin{aligned}
\frac{\sigma}{\lambda T} & -\left(5 M+\frac{\sigma}{2}\right) \frac{\partial \beta_{\epsilon}}{\partial s}\left(x_{0}-y_{0}, t_{0}-s_{0}\right) \\
& -g\left(\left(5 M+\frac{\sigma}{2}\right) \nabla_{y} \beta_{\epsilon}\left(x_{0}-y_{0}, t_{0}-s_{0}\right)\right) \leqslant 0 .
\end{aligned}
$$

Adding (3.2.1) and (3.2.2) gives

$$
\begin{aligned}
\frac{2 \sigma}{\lambda T} & -\left(5 M+\frac{\sigma}{2}\right) \frac{\partial \beta_{\epsilon}}{\partial t}\left(x_{0}-y_{0}, t_{0}-s_{0}\right)-\left(5 M+\frac{\sigma}{2}\right) \frac{\partial \beta_{\epsilon}}{\partial s}\left(x_{0}-y_{0}, t_{0}-s_{0}\right) \\
& +f\left(-\left(5 M+\frac{\sigma}{2}\right) \nabla_{x} \beta_{\epsilon}\left(x_{0}-y_{0}, t_{0}-s_{0}\right)\right) \\
& -g\left(\left(5 M+\frac{\sigma}{2}\right) \nabla_{y} \beta_{\epsilon}\left(x_{0}-y_{0}, t_{0}-s_{0}\right)\right) \leqslant 0 .
\end{aligned}
$$

Since $\left.\frac{\partial \beta_{\epsilon}}{\partial t}\left(x_{0}-y_{0}, t-s_{0}\right)\right|_{t=t_{0}}=-\left.\frac{\partial \beta_{\epsilon}}{\partial s}\left(x_{0}-y_{0}, t_{0}-s\right)\right|_{s=s_{0}}$ and $\nabla_{x} \beta_{\varepsilon}\left(x-y_{0}, t_{0}-s_{0}\right)$ $\left.\right|_{x=x_{0}}=-\left.\nabla_{y} \beta_{\epsilon}\left(x_{0}-y, t_{0}-s_{0}\right)\right|_{y=y_{0}}$, we have

$$
\frac{2 \sigma}{\lambda T}+(f-g)\left(-\left(5 M+\frac{\sigma}{2}\right) \nabla_{x} \beta_{\epsilon}\left(x_{0}-y_{0}, t_{0}-s_{0}\right)\right) \leqslant 0 .
$$

Hence

$$
\begin{aligned}
& \frac{2 \sigma}{\lambda T} \leqslant(g-f)\left(-\left(5 M+\frac{\sigma}{2}\right) \nabla_{x} \beta_{\epsilon}\left(x_{0}-y_{0}, t_{0}-s_{0}\right)\right) \\
\Rightarrow & \frac{2 \sigma}{\lambda T} \leqslant\|g-f\|_{L^{\infty}\left(\mathbb{R}^{N}\right)} \\
\Rightarrow & \sigma \leqslant \frac{\lambda T}{2}\|f-g\|_{L^{\infty}\left(\mathbb{R}^{N}\right)} .
\end{aligned}
$$


Letting $\lambda \rightarrow 2$, we obtain

$$
\sigma \leqslant T\|f-g\|_{L^{\infty}\left(\mathbb{R}^{N}\right)}
$$

We now drop the assumption (A). For $r>0$, let $\rho(x)$ be a smooth function having support in the ball $B(0, r+1)=\left\{x \in \mathbb{R}^{N}|| x \mid \leqslant r+1\right\}$ such that $\rho(x)=1$ on $|x| \leqslant r$. Suppose that $u_{0}^{\rho}(x)=\rho(x) u_{0}(x)$ and $w_{0}^{\rho}(x)=\rho(x) w_{0}(x)$, then for all positive time $t$, the corresponding viscosity solutions $u^{\rho}(x)$ and $w^{\rho}(x)$ have the following properties:

$$
\begin{aligned}
& u^{\rho}(x)=u(x) \text { on }|x|<r-t|f|_{\text {Lip }} \text { and } \\
& w^{\rho}(x)=w(x) \text { on }|x|<r-t|g|_{\text {Lip }} \text {; }
\end{aligned}
$$

see $[11]$. Let $L=\max \left\{|f|_{\text {Lip }},|g|_{\text {Lip }}\right\}$. Then

$$
\begin{aligned}
\max _{|x|<r-L t}|u(x, t)-w(x, t)| & =\max _{|x|<r-L t}\left|u^{\rho}(x, t)-w^{\rho}(x, t)\right| \\
& \leqslant t\|f-g\|_{L^{\infty}\left(\mathbb{R}^{N}\right)} \quad \text { by the previous argument. }
\end{aligned}
$$

Hence, letting $r \rightarrow \infty$, we have

$$
\|u-w\|_{L^{\infty}\left(\mathbb{R}^{N}\right)} \leqslant t\|f-g\|_{L^{\infty}\left(\mathbb{R}^{N}\right)}
$$

This completes the proof.

We now prove Theorem 3.1 .

Proof of Theorem 3.1: In addition to the equation in the statement of Theorem 3.1 , consider

$$
\begin{array}{ll}
w_{t}+g(\nabla w)=0, & x \in \mathbb{R}^{N}, t>0, \\
w(x, 0)=u_{0}(x), & x \in \mathbb{R}^{N} .
\end{array}
$$

Then, by Lemma 3.2 and Lemma 2.3,

$$
\begin{aligned}
& \|u(\cdot, t)-v(\cdot, t)\|_{L^{\infty}\left(\mathbb{R}^{N}\right)} \\
& \quad \leqslant\|u(\cdot, t)-w(\cdot, t)\|_{L^{\infty}\left(\mathbb{R}^{N}\right)}+\|w(\cdot, t)-v(\cdot, t)\|_{L^{\infty}\left(\mathbb{R}^{N}\right)} \\
& \quad \leqslant t\|f-g\|_{L^{\infty}\left(\mathbb{R}^{N}\right)}+\|w(\cdot, 0)-v(\cdot, 0)\|_{L^{\infty}\left(\mathbb{R}^{N}\right)} \\
& \quad=\|f-g\|_{L^{\infty}\left(\mathbb{R}^{N}\right)}+\left\|u_{0}-v_{0}\right\|_{L^{\infty}\left(\mathbb{R}^{N}\right)} .
\end{aligned}
$$

This completes the proof. 


\section{Approximation spaces, Besov spaces and Besov-type spaces}

In this section we give the definitions of approximation spaces, Besov spaces, and Besov-type spaces, and we recall the relationship between approximation spaces and Besov-type spaces. The general references for this section are [7] and [13].

Consider the approximation of functions in $C(I)$ where $I:=[0,1]$. For any $f \in$ $C(I)$ and any positive integer $N$, let $E_{N}^{3}(f, C(I)):=\inf \|f-\phi\|_{C(I)}$ where the infimum is taken over all continuous, piecewise polynomial functions $\phi$ defined on $I$ of degree less than 3 with $N-1$ free interior knots. For each positive $\alpha$ less than 3 and $q \in(0, \infty]$, define $A_{q}^{\alpha}(C(I))$ to be the set of functions for which

$$
\|f\|_{A_{q}^{\alpha}(C(I))}:=\|f\|_{C(I)}+\left(\sum_{N=1}^{\infty}\left[N^{\alpha} E_{N}^{3}(f, C(I))\right]^{q} \frac{1}{N}\right)^{1 / q}<\infty
$$

if $0<q<\infty$ and

$$
\|f\|_{A_{q}^{\alpha}(C(I))}:=\|f\|_{C(I)}+\sup _{N}\left\{N^{\alpha} E_{N}^{3}(f, C(I))\right\}<\infty
$$

if $q=\infty$.

REMARK. If $\alpha_{1}>\alpha_{2}$, then $A_{q_{1}}^{\alpha_{1}}(C(I)) \subset A_{q_{2}}^{\alpha_{2}}(C(I))$ for any $q_{1}$ and $q_{2}$. But if $\alpha_{1}=$ $\alpha_{2}=\alpha$ and $q_{1}>q_{2}$, then $A_{\dot{q}_{1}}^{\alpha}(C(I)) \supset A_{q_{2}}^{\alpha}(C(I))$.

We now define Besov spaces. For $\alpha \in(0, \infty), q \in(0, \infty]$ and $p \in(0, \infty]$, the Besov spaces $B_{q}^{\alpha}\left(L^{p}(I)\right)$ are defined as follows: Pick any integer $r>\alpha$, let $\nabla^{r}(f, h)(x)$ be the $r$ th forward difference of $f$ at $x$ with step $h$ (that is, $\nabla^{0}(f, h)(x):=f(x)$ and $\left.\nabla^{r}(f, h)(x):=\nabla^{r-1}(f, h)(x+h)-\nabla^{r-1}(f, h)(x)\right)$. Let $I_{r h}=\{x \in I \mid x+r h \in I\}$. Define $\omega_{r}(f, t)_{p}=\sup _{|h|<t}\left\|\nabla^{r}(f, h)\right\|_{L^{p}\left(I_{r h}\right)}$. The Besov space $B_{q}^{\alpha}\left(L^{p}(I)\right)$ is defined to be the set of functions $f$ for which

$$
|f|_{B_{q}^{\alpha}\left(L^{p}(I)\right)}:=\left(\int_{0}^{\infty}\left[t^{-\alpha} \omega_{r}(f, t)_{p}\right]^{q} \frac{d t}{t}\right)^{1 / q}<\infty
$$

if $0<q<\infty$ and

$$
|f|_{B_{q}^{\alpha}\left(L^{p}(I)\right)}:=\sup _{0<t<\infty}\left\{t^{-\alpha} \omega_{r}(f, t)_{p}\right\}<\infty
$$

if $q=\infty$. Set $\|f\|_{B_{q}^{\alpha}\left(L^{P}(I)\right)}:=\|f\|_{L^{P}(I)}+|f|_{B_{q}^{\alpha}\left(L^{p}(I)\right)}$.

The relationship between approximation spaces and Besov spaces can be shown by means of $K$-functionals. The real method of interpolation using $K$-functionals can be described as follows. For any two spaces $X_{0}$ and $X_{1}$ contained in a linear metric space $X$, define the following functional for all $f$ in $X_{0}+X_{1}$.

$$
K\left(f, t, X_{0}, X_{1}\right):=\inf _{f=f_{0}+f_{1}}\left\{\left\|f_{0}\right\|_{X_{0}}+t\left\|f_{1}\right\|_{X_{1}}\right\}
$$


where $f_{0} \in X_{0}$ and $f_{1} \in X_{1}$. The new space $X_{\theta, q}=\left(X_{0}, X_{1}\right)_{\theta, q}(0<\theta<1,0<q \leqslant \infty)$ consists of functions $f$ for which

$$
\|f\|_{\theta, q}:=\|f\|_{X_{0}+X_{1}}+\left(\int_{0}^{\infty}\left[t^{-\theta} K\left(f, t, X_{0}, X_{1}\right)\right]^{q} \frac{d t}{t}\right)^{1 / q}<\infty,
$$

where $\|f\|_{X_{0}+X_{1}}=K\left(f, 1, X_{0}, X_{1}\right)$.

The approximation spaces $A_{q}^{\alpha}(C(I))$ cannot in general be characterised in terms of Besov spaces. It is possible however to characterise $A_{q}^{\alpha}(C(I))$ in terms of certain spaces which are closely related to Besov spaces. For this type of approximation, Petrushev [13] has given Jackson and Bernstein inequalities in terms of the space $B_{*}^{\alpha}, 3>\alpha>1$, consisting of all absolutely continuous functions $f$ for which $f^{\prime}$ is in $B_{q}^{\alpha-1}\left(L^{q}(I)\right)$ where $\alpha=1 / q$. That is, $E_{N}^{3}\left(u_{0}, C(I)\right) \leqslant C N^{-\alpha}\left\|u_{0}\right\|_{B_{*}^{\alpha}}$ (the Jackson inequality) and $\|\phi\|_{B^{\alpha}} \leqslant C N^{\alpha}\|\phi\|_{C}$ (the Bernstein inequality) for any continuous, piecewise polynomial $\phi$ of degree less than 3 . (Petrushev in fact proved such inequalities for polynomials of degree less than $r$ and $1<\alpha<r$.) The first statement of the following theorem follows directly from these estimates, while the second statement relies on a characterisation of some of these interpolation spaces by DeVore and Popov [7].

THEOREM 4.1. When $0<q \leqslant \infty, 1<\beta \leqslant 3$ and $1<\alpha<\beta$,

$$
A_{q}^{\alpha}(C(I))=\left(C(I), B_{*}^{\beta}\right)_{\alpha / \beta, q}
$$

and if $q=1 / \alpha$,

$$
A_{q}^{\alpha}(C(I))=B_{*}^{\alpha} .
$$

\section{REGULARITY FOR HAMILTON-JACOBI EQUATIONS}

We prove the following theorem and corollary that are the main results of this paper. A similar result for conservation laws was shown by DeVore and Lucier [6].

THEOREM 5.1. Suppose that $u_{0}$ is Lipschitz continuous, has support in $[0,1]$, and that $u_{0}^{\prime} \in B V(\mathbb{R})$. Let $\alpha \in(0,3)$ and $q \in(0, \infty]$ and let $\Omega=\{y|| y \mid \leqslant$ $\left.C\left|u_{0}^{\prime}\right|_{\mathrm{BV}(\mathbb{I})}\right\}$, where $C$ will be specified latter. If $f(0)=0, f^{\prime \prime}>0$, and $f^{\prime}$ and $f^{\prime \prime \prime}$ are bounded on $\Omega$, and if $u_{0} \in A_{q}^{\alpha}(C([0,1]))$, then for all $t>0, u(\cdot, t)$ has support in $I_{t}=\left[\inf _{\rho \in \Omega} f^{\prime}(\rho) t, 1+\sup _{\rho \in \Omega} f^{\prime}(\rho) t\right]$, is Lipschitz continuous, and is in $A_{q}^{\alpha}\left(C\left(I_{t}\right)\right)$.

Corollary 5.1. Let $\alpha \in(1,3)$. Suppose that $u_{0}$ is Lipschitz continuous, has support in $[0,1]$ and that $u_{0}^{\prime} \in B V(\mathbb{R})$. Let $f(0)=0$. Suppose that $f^{\prime \prime}>0$ and that $f^{\prime}$ and $f^{\prime \prime \prime}$ are bounded on $\Omega$ as given in Theorem 5.1. If $u_{0}$ is in $B_{*}^{\alpha}([0,1])$, then $u(\cdot, t)$ has support in $I_{t}$ given in Theorem 5.1 and is in $B_{*}^{\alpha}\left(I_{t}\right)$ for all $t>0$.

Suppose that $v_{0}$ is a good continuous, piecewise quadratic approximation to $u_{0}$ with $N-1$ free interior knots where $I:=[0,1]$ such that $\left\|u_{0}-v_{0}\right\|_{C(I)} \leqslant$ 
$2 \inf \left\|u_{0}-\phi\right\|_{C(I)}=2 E_{N}^{3}\left(u_{0}, C(I)\right)$ and that $v_{0}$ has support in $I$. Let $\left\{\tau_{i}\right\}_{i=0}^{N}$, with $\tau_{0}=0$ and $\tau_{N}=1$, be the ordered set of knots of $v_{0}$. Consider each interval $I_{i}:=\left[\tau_{i}, \tau_{i+1}\right]$. Now $\left|I_{i}\right|:=\tau_{i+1}-\tau_{i}$, and let $P_{i}$ be a quadratic approximation to $u_{0}$ on each $I_{i}$ such that $\left\|u_{0}-P_{i}\right\|_{C\left(I_{i}\right)} \leqslant\left\|u_{0}-v_{0}\right\|_{C(I)}$. Let $z_{0}(x)$ be the piecewise, discontinuous quadratic approximation to $u_{0}$ satisfying $\left.z_{0}\right|_{I_{i}}=P_{i}$ for $i=0, \ldots, N-1$ and having support in $I$. Then $\left\|u_{0}-z_{0}\right\|_{Z^{\infty}(\mathbb{R})} \leqslant\left\|u_{0}-v_{0}\right\|_{C(\mathbb{R})}$.

Now construct a continuous, piecewise quadratic approximation $w_{0}(x)$ having support in $[0,1]$ from $z_{0}(x)$ as follows. On each interval $I_{i}$, add a linear polynomial $l_{i}(x)$ to $P_{i}(x)$ so that $w_{0}\left(\tau_{i}\right)=u_{0}\left(\tau_{i}\right)$ and $w_{0}\left(\tau_{i+1}\right)=u_{0}\left(\tau_{i+1}\right)$. Then $\left\|l_{i}(x)\right\|_{L^{\infty}\left(I_{i}\right)} \leqslant\left\|u_{0}-v_{0}\right\|_{C(\mathbb{R})}$ for all $i$. Hence

$$
\begin{aligned}
\left\|u_{0}-w_{0}\right\|_{C(\mathbb{R})} & \leqslant\left\|u_{0}-z_{0}\right\|_{L^{\infty}(\mathbb{R})}+\left\|z_{0}-w_{0}\right\|_{L^{\infty}(\mathbb{R})} \\
& \leqslant\left\|u_{0}-v_{0}\right\|_{C(\mathbb{B})}+\max _{i}\left\|l_{i}(x)\right\|_{L^{\infty}\left(I_{i}\right)} \\
& \leqslant 2\left\|u_{0}-v_{0}\right\|_{C(\mathbb{R})} \\
& \leqslant 4 E_{N}^{3}\left(u_{0}, C(I)\right) .
\end{aligned}
$$

Let $\eta_{0}(x)$ be the piecewise linear interpolant to $u_{0}(x)$ at $\left\{\tau_{i}\right\}_{i=1}^{N}$. Then we have the following lemmas.

LEMma 5.2. If $u_{0}^{\prime} \in B V(\mathbb{R})$, then $\left|\eta_{0}^{\prime}\right|_{\mathrm{BV}(\mathbb{R})} \leqslant\left|u_{0}^{\prime}\right|_{\mathrm{BV}(\mathbb{R})}$.

Proof: One can see that for each $i$,

$$
\eta_{0}^{\prime} \mid I_{i}=\frac{1}{\left|I_{i}\right|} \int_{I_{i}} u_{0}^{\prime} d x
$$

For each $i$, one can find $\bar{\sigma}_{i}, \underline{\sigma}_{i} \in\left[\tau_{i}, \tau_{i+1}\right]$ such that $u_{0}^{\prime}\left(\underline{\sigma}_{i}\right) \leqslant\left.\eta_{0}^{\prime}\right|_{I_{i}} \leqslant u_{0}^{\prime}\left(\bar{\sigma}_{i}\right)$. We now order them. Then we have at most $2 N$ distinct knots, say $\zeta_{i}$ for $i=1, \ldots, 2 N$. Hence,

$$
\begin{aligned}
\left|\eta_{0}^{\prime}\right|_{\mathrm{BV}(\mathbb{R})}= & \sum_{i=0}^{N-2}\left|\eta_{0}^{\prime}\right|_{I_{i}}-\eta_{0}^{\prime}\left|{ }_{I_{i+1}}\right| \\
& +\left|\eta_{0}^{\prime}\right|_{I_{0}}|+| \eta_{0}^{\prime}\left|I_{N-1}\right| \\
\leqslant & \sum_{i=1}^{2 N-1}\left|u_{0}^{\prime}\left(\zeta_{i}\right)-u_{0}^{\prime}\left(\zeta_{i+1}\right)\right| \\
& +\left|u_{0}^{\prime}\left(\zeta_{1}\right)\right|+\left|u_{0}^{\prime}\left(\zeta_{2 N}\right)\right| \\
\leqslant & \left|u_{0}^{\prime}\right|_{\mathrm{BV}(\mathbf{I})} .
\end{aligned}
$$

This completes the prorif. 
Lemma 5.3. Suppose that $u_{0}^{\prime} \in B V(\mathbb{R})$ with support in $[0,1]$. Then there is a constant $C$ such that the approximation $w_{0}$ satisfies $\left|w_{0}^{\prime}\right|_{\mathrm{BV}(\mathbb{B})} \leqslant C\left|u_{0}^{\prime}\right|_{\mathrm{BV}(\mathbb{B})} \cdot$

Proof: Let $L_{i}(x)$ be the linear polynomial on each $I_{i}$ satisfying $L_{i}\left(\tau_{i}\right)=u_{0}\left(\tau_{i}\right)$ and $L_{i}^{\prime}\left(\tau_{i}\right)=u_{0}^{\prime}\left(\tau_{i}\right)$ for each $i$. (Here we assume that $u_{0}^{\prime}$ is taken to be right continuous.) Since $w_{0}^{\prime \prime}$ is a constant and $u_{0}^{\prime \prime}$ is a measure,

$$
\begin{aligned}
\int_{I_{i}}\left|w_{0}^{\prime \prime}\right| d x & =\int_{I_{i}}\left|z_{0}^{\prime \prime}\right| d x \\
& =\int_{I_{i}}\left|z_{0}^{\prime \prime}-L_{i}^{\prime \prime}\right| d x \\
& \leqslant \frac{C}{\left|I_{i}\right|}\left\|z_{0}-L_{i}\right\|_{L^{\infty}\left(I_{i}\right)} \quad \text { (true for polynomials of fixed degree) } \\
& \leqslant \frac{C}{\left|I_{i}\right|}\left\{\left\|z_{0}-u_{0}\right\|_{L^{\infty}\left(I_{i}\right)}+\left\|u_{0}-L_{i}\right\|_{L^{\infty}\left(I_{i}\right)}\right\} \\
& \leqslant \frac{C}{\left|I_{i}\right|}\left\|u_{0}-L_{i}\right\|_{L^{\infty}\left(I_{i}\right)} \\
& =\frac{C}{\left|I_{i}\right|} \sup _{x \in I_{i}}\left|u_{0}(x)-L_{i}(x)\right| \\
& =\frac{C}{\left|I_{i}\right|} \sup _{x \in I_{i}}\left|u_{0}\left(\tau_{i}\right)+u_{0}^{\prime}\left(\tau_{i}\right)\left(x-\tau_{i}\right)+\int_{\tau_{i}}^{x}(x-s) u_{0}^{\prime \prime}(s) d s-L_{i}(x)\right| \\
& \leqslant \frac{C}{\left|I_{i}\right|} \sup _{x \in I_{i}} \int_{\tau_{i}}^{x}|x-s|\left|u_{0}^{\prime \prime}\right| d x \\
& \leqslant \frac{C}{\left|I_{i}\right|}\left|I_{i}\right| \int_{I_{i}}\left|u_{0}^{\prime \prime}\right| d s \\
& =C \int_{I_{i}}\left|u_{0}^{\prime \prime}\right| d s .
\end{aligned}
$$

Hence $\left|w_{0}^{\prime}\right|_{\mathrm{BV}\left(I_{i}\right)} \leqslant C\left|u_{0}^{\prime}\right|_{\mathrm{BV}\left(I_{i}\right)}$ for all $i$.

We now measure the jump $\left|w_{0}^{\prime}\left(\tau_{i}^{+}\right)-w_{0}^{\prime}\left(\tau_{i}^{-}\right)\right|$. Because $w_{0}$ is quadratic on $I_{i}$, $\eta_{0}$ is linear on $I_{i}$, and $w_{0}\left(\tau_{i}\right)=\eta_{0}\left(\tau_{i}\right)$ for all $i$, one can see that

Therefore,

$$
\left|w_{0}^{\prime}\left(\tau_{i}^{+}\right)-\eta_{0}^{\prime}\left(\tau_{i}^{+}\right)\right|=\frac{1}{2} \int_{\tau_{i}}^{\tau_{i+1}}\left|w_{0}^{\prime \prime}(x)\right| d x \leqslant \frac{C}{2} \int_{\tau_{i}}^{\tau_{i+1}}\left|u_{0}^{\prime \prime}(x)\right| d x .
$$

$$
\begin{aligned}
\sum_{i=0}^{N}\left|w_{0}^{\prime}\left(\tau_{i}^{+}\right)-w_{0}^{\prime}\left(\tau_{i}^{-}\right)\right| \leqslant & \sum_{i=0}^{N}\left|\eta_{0}^{\prime}\left(\tau_{i}^{+}\right)-\eta_{0}^{\prime}\left(\tau_{i}^{-}\right)\right|+\sum_{i=0}^{N}\left|w_{0}^{\prime}\left(\tau_{i}^{+}\right)-\eta_{0}^{\prime}\left(\tau_{i}^{+}\right)\right| \\
& +\sum_{i=0}^{N}\left|w_{0}^{\prime}\left(\tau_{i}^{-}\right)-\eta_{0}^{\prime}\left(\tau_{i}^{-}\right)\right| \\
\leqslant & (1+C)\left|u_{0}^{\prime}\right|_{\mathrm{BV}(\mathrm{B})} .
\end{aligned}
$$


Adding these two constants gives us the desired value of $C$.

This $C$ is the constant of Theorem 5.1. Since $C$ is greater than 1 and $\left|w_{0}^{\prime}\right|_{\mathrm{BV}(\mathbb{I})} \leqslant$ $C\left|u_{0}^{\prime}\right|_{\mathrm{BV}(\mathbb{R})},\left\|w_{0}^{\prime}\right\|_{L^{\infty}(\mathbb{R})} \leqslant\left|w_{0}^{\prime}\right|_{\mathrm{BV}(\mathbb{R})} \leqslant C\left|u_{0}^{\prime}\right|_{\mathrm{BV}(\mathbb{I})}$ and $\left\|u_{0}^{\prime}\right\|_{L^{\infty}(\mathbb{R})} \leqslant\left|u_{0}^{\prime}\right|_{\mathrm{BV}(\mathbb{R})} \leqslant$ $C\left|u_{0}^{\prime}\right|_{\mathrm{BV}(\mathbb{B})}$. So, the ranges of $u_{0}^{\prime}$ and $w_{0}^{\prime}$ are contained in $\Omega=\left\{\left.y|| y|\leqslant C| u_{0}^{\prime}\right|_{\mathrm{BV}(\mathbb{R})}\right\}$.

There is a $C^{1}$ piecewise quadratic approximation $f_{\epsilon}$ to $f$ with knots at the points $j / N, j \in \mathbb{Z}$, that is defined by $f^{\prime}(j / N)=f_{\epsilon}^{\prime}(j / N), f(0)=f_{\epsilon}(0) ; f_{\epsilon}$ is strictly convex. Moreover $\left\|f-f_{\epsilon}\right\|_{L^{\infty}(\mathbb{R})} \leqslant C\left\|f^{\prime \prime \prime}\right\|_{L^{\infty}(\mathbb{I})} N^{-3}$; see $[1,5]$. Consider now the perturbed problem

$$
w_{t}+f_{\epsilon}\left(w_{x}\right)=0, \quad x \in \mathbb{R}, t>0,
$$

$$
w(x, 0)=w_{0}(x), \quad x \in \mathbb{R} .
$$

Lions [11] proved that the viscosity solution of this equation can be found using a technique introduced by $\operatorname{Lax}[\mathbf{1 0}]$, which we shall now describe. Let $z=f_{\epsilon}^{\prime}(w)$ and $g(z)=z w-f_{\epsilon}(w)$. Then $g(z)=z\left(f_{\epsilon}^{\prime}\right)^{-1}(z)-f_{\epsilon}\left(\left(f_{\epsilon}^{\prime}\right)^{-1}(z)\right)$ and

$$
\begin{aligned}
\frac{d g}{d z} & =\left(f_{\epsilon}^{\prime}\right)^{-1}(z)+z\left(\left(f_{\epsilon}^{\prime}\right)^{-1}\right)^{\prime}(z)-f_{\epsilon}^{\prime}\left(\left(f_{\epsilon}^{\prime}\right)^{-1}(z)\right)\left(\left(f_{\epsilon}^{\prime}\right)^{-1}\right)^{\prime}(z) \\
& =\left(f_{\epsilon}^{\prime}\right)^{-1}(z)+z\left(\left(f_{\epsilon}^{\prime}\right)^{-1}\right)^{\prime}(z)-z\left(\left(f_{\epsilon}^{\prime}\right)^{-1}\right)^{\prime}(z) \\
& =\left(f_{\epsilon}^{\prime}\right)^{-1}(z) \\
& =w .
\end{aligned}
$$

But $\left(f_{\epsilon}^{\prime}\right)^{-1}$ is increasing, so $g(z)$ is strictly convex. Moreover, $g^{\prime}$ and $f_{\epsilon}^{\prime}$ are inverse functions.

Lions [11] showed that $w(x, t)=\min _{y \in \mathbb{B}}\left\{t g((x-y) / t)+w_{0}(y)\right\}$ is the viscosity solution of $(\mathrm{P})$, and $w(x, t)$ is Lipschitz continuous if $w_{0}$ is Lipschitz continuous and $f_{\epsilon}$ is convex. The minimisation problem introduced by Lax [10] picks out a specific value $y:=y(x, t)$ among possibly many solutions of $(x-y) / t=f_{\epsilon}^{\prime}\left(w_{0}^{\prime}(y)\right)$ so that $w(x, t)=\min _{y \in \mathbb{R}}\left\{t g((x-y) / t)+w_{0}(y)\right\}$ is attained. Moreover, he shows that $y(x, t)$ is an increasing function of $x$ for fixed $t$. The derivative of the solution $w(x, t)$ with respect to $x$ is discontinuous wherever $y(x, t)$ is discontinuous in $x$.

We now count some points, which we call points of transition, at which the derivative of the viscosity solution $w(x, t)$ with respect to $x$ is not continuous. We fix $t$ and introduce 2 types of points in $I:=[0,1]$.

The first are the knots of $w_{0}(x)$. By construction, there are $N$ such points. 
The second type of points are isolated points $x$ where $w_{0}^{\prime}(x)=j / N$ for some $j \in \mathbb{Z}$. If $w_{0}^{\prime}$ is discontinuous at $x$ and there are several values of $j$ such that $\min \left(w_{0}^{\prime}\left(x^{-}\right), w_{0}^{\prime}\left(x^{+}\right)\right)<j / N<\max \left(w_{0}^{\prime}\left(x^{+}\right), w_{0}^{\prime}\left(x^{-}\right)\right)$, then add these points to the second type. On each interval $I_{i}=\left[\tau_{i}, \tau_{i+1}\right]$, the number of isolated points for $w_{0}^{\prime}$ is no more than $N\left|w_{0}^{\prime}\right|_{\mathbf{B V}\left(I_{i}\right)}+1$ by the following argument. Since $w_{0}^{\prime}(x)$ is linear on $I_{i}$, the number of isolated points for $w_{0}^{\prime}(x)$ is the same as the number of values of $j$ for which $j / N$ is on the interval $\left[\min \left(w_{0}^{\prime}\left(\tau_{i}^{+}\right), w_{0}^{\prime}\left(\tau_{i+1}^{-}\right)\right), \max \left(w_{0}^{\prime}\left(\tau_{i}^{+}\right), w_{0}^{\prime}\left(\tau_{i+1}^{-}\right)\right)\right]$ and the number of these points is at most $N$ times the length of the interval $\left[\min \left(w_{0}^{\prime}\left(\tau_{i}^{+}\right), w_{0}^{\prime}\left(\tau_{i+1}^{-}\right)\right), \max \left(w_{0}^{\prime}\left(\tau_{i}^{+}\right), w_{0}^{\prime}\left(\tau_{i+1}^{-}\right)\right)\right]$plus 1 which is $N\left|w_{0}^{\prime}\right|_{\mathrm{BV}\left(I_{i}\right)}+1$. So, the total number of isolated points is no more than $\sum_{i=0}^{N-1}\left(N\left|w_{0}^{\prime}\right|_{\mathrm{BV}\left(I_{i}\right)}+1\right)$. Similarly, when $w_{0}^{\prime}(x)$ is discontinuous at $x$, the number of values of $j$ satisfying $\min \left(w_{0}^{\prime}\left(x^{-}\right), w_{0}^{\prime}\left(x^{+}\right)\right)<j / N<\max \left(w_{0}^{\prime}\left(x^{+}\right), w_{0}^{\prime}\left(x^{-}\right)\right)$is at most $N\left|w_{0}^{\prime}\left(\tau_{i}^{+}\right)-w_{0}^{\prime}\left(\tau_{i}^{-}\right)\right|$. So, the total number of these points is at most. $N \sum_{i=0}^{N}\left|w_{0}^{\prime}\left(\tau_{i}^{+}\right)-w_{0}^{\prime}\left(\tau_{i}^{-}\right)\right|$. Overall, the total number of points of the second type is, by Lemma 5.2, no more than $\left(1+2 C\left|u_{0}^{\prime}\right|_{\mathrm{BV}(\mathbb{R})}\right) N$ because $\sum_{i=1}^{N}\left(N\left|w_{0}^{\prime}\right|_{\mathrm{BV}\left(I_{i}\right)}+1\right) \leqslant\left(1+C\left|u_{0}^{\prime}\right|_{\mathrm{BV}(\mathbb{R})}\right) N$ and $N \sum_{i=0}^{N}\left|w_{0}^{\prime}\left(\tau_{i}^{+}\right)-w_{0}^{\prime}\left(\tau_{i}^{-}\right)\right| \leqslant C\left|u_{0}^{\prime}\right|_{\mathrm{BV}(\mathbb{B})} N$. Consider $F(y(x, t))=y(x, t)+$ $t f_{\epsilon}^{\prime}\left(w_{0}^{\prime}(y(x, t))\right)$. Since $F(y)$ is a linear polynomial in $y$ between points of type one and type two, there is no zero of $F^{\prime}(y)$ between points of type one and type two. Therefore, $F(y)$ is monotone on each open interval which contains no points of the two types described above. The solution $w(x, t)$ of (P) satisfies $w(x, t)=g((x-y) / t)+w_{0}(y)$ for some solution $y:=y(x, t)$ to the equation

$$
\frac{x-y}{t}=f_{\epsilon}^{\prime}\left(w_{0}^{\prime}(y)\right) \text {. }
$$

Consider now a maximal interval $I_{0}$ of $x$ values on which $y$ takes values in an interval $J$ which contains no points of the two types described above. Since $F(y)$ is monotone on $J$, there is at most one solution of $y$ to (5.1) in $J$. Since $y$ increases as $x$ increases, each interval $I_{0}$ contains at most one point at which the jump of $y(x, t)$ happens. (That is, the derivative of $w(x, t)$ is discontinuous.) Since there are at most $\left(2+2 C\left|u_{0}^{\prime}\right|_{\mathrm{BV}(\mathbb{R})}\right) N$ intervals $I_{0}$, there are at most $\left(2+2 C\left|u_{0}^{\prime}\right|_{\mathrm{BV}(\mathbb{R})}\right) N$ points of transition in the solution $w(x, t)$. Between these points of transition, we can easily see that

$$
w_{x}(x, t)=g^{\prime}\left(\frac{x-y}{t}\right)
$$

Since $g^{\prime}(s)$ is linear in $s$ and $y$ is linear in $x, w_{x}(x, t)$ is linear in $x$. So, $w(x, t)$ is quadratic in $x$ between any two points of transition. Therefore, the solution $w(x, t)$ 
of the perturbed problem (P) is a continuous, piecewise quadratic polynomial for fixed $t>0$ with no more than $\left(2+2 C\left|u_{0}^{\prime}\right|_{\mathrm{BV}(\mathbb{R})}\right) N$ pieces.

THEOREM 5.4. Let $u_{0}^{\prime} \in B V(\mathbb{R})$ and let $u_{0}$ have support in $I:=[0,1]$. Let $f(0)=0$. Suppose that $f^{\prime \prime}>0$ and that $f^{\prime}$ and $f^{\prime \prime \prime}$ are bounded on $\Omega$ as defined in Theorem 5.1. Suppose that $\alpha \in(0,3)$ and $u_{0} \in A_{q}^{\alpha}(C(I))$ where $q \in(0, \infty]$. Then $u(\cdot, t)$ has support in $I_{t}=\left[\inf _{\rho \in \Omega} t f^{\prime}(\rho), \sup _{\rho \in \Omega} 1+t f^{\prime}(\rho)\right]$ and for any $N \geqslant 1$,

$$
E_{F(N)}^{3}\left(u(\cdot, t), L^{\infty}\left(I_{t}\right)\right) \leqslant 4 E_{N}^{3}\left(u_{0}, L^{\infty}(I)\right)+\frac{C_{1} t}{N^{3}}\left\|f^{\prime \prime \prime}\right\|_{L^{\infty}(\mathbb{R})},
$$

where $F(N)=\left(2+2 C\left|u_{0}^{\prime}\right|_{\mathrm{BV}(\mathbb{R})}\right) N$ and $C$ is given in Lemma 5.3.

PROOF: The first conclusion is classical. We shall prove the second conclusion. Consider two equations

$$
\begin{array}{ll}
u_{t}+f\left(u_{x}\right)=0, & x \in \mathbb{R}, t>0, \\
u(x, 0)=u_{0}(x), & x \in \mathbb{R},
\end{array}
$$

and

$$
\begin{array}{ll}
w_{t}+f_{\epsilon}\left(w_{x}\right)=0, & x \in \mathbb{R}, t>0, \\
w(x, 0)=w_{0}(x), & x \in \mathbb{R} .
\end{array}
$$

By Theorem 3.1 and the construction of $f_{\varepsilon}$,

$$
\begin{aligned}
\|u(\cdot, t)-w(\cdot, t)\|_{L^{\infty}(\mathbb{R})} & \leqslant\left\|u_{0}-v_{0}\right\|_{L^{\infty}(\mathbb{R})}+f\left\|f-f_{\epsilon}\right\|_{L^{\infty}(\mathbb{R})} \\
& \leqslant\left\|u_{0}-w_{0}\right\|_{L^{\infty}(\mathbb{R})}+\frac{C_{1} t}{N^{3}}\left\|f^{\prime \prime \prime}\right\|_{L^{\infty}(\mathbb{R})} .
\end{aligned}
$$

Since $\left\|u_{0}-w_{0}\right\|_{L^{\infty}(\mathbb{R})} \leqslant 2\left\|u_{0}-v_{0}\right\|_{L^{\infty}(\mathbb{R})} \leqslant 4 E_{N}^{3}\left(u_{0}, C(I)\right), w(\cdot, t)$ has no more than $F(N)$ breakpoints, and $E_{F(N)}^{3}\left(u, C\left(I_{t}\right)\right) \leqslant\|u(\cdot, t)-w(\cdot, t)\|_{L^{\infty}(\mathbb{R})}$, the second conclusion immediately follows.

Theorem 5.4 can be now used to prove Theorem 5.1.

Proof of Theorem 5.1: Inequality (5.4) shows that

$$
E_{N}^{3}\left(u(\cdot, t) C\left(I_{t}\right)\right) \leqslant 4 E_{C_{2} N}^{3}\left(u_{0}, C(I)\right)+C_{3} / N^{3},
$$

where $C_{2}=1 /\left(2+2 C\left|u_{0}^{\prime}\right|_{\mathrm{BV}(\mathbb{R})}\right)$ and $C_{3}=C_{1} t\left\|f^{\prime \prime \prime}\right\|_{L^{\infty}(\mathbb{R})} /\left(2+2 C\left|u_{0}^{\prime}\right|_{\mathrm{BV}(\mathbb{I})}\right)^{3}$. 
From now on, we shall use a generic $C$. For $0<q<\infty$,

$$
\begin{aligned}
& \left(\sum_{N=1}^{\infty}\left[N^{\alpha} E_{N}\left(u(\cdot, t), C\left(I_{t}\right)\right)\right]^{q} \frac{1}{N}\right)^{1 / q} \\
& \quad \leqslant\left(\sum_{N=1}^{\infty}\left[N^{\alpha}\left(C E_{C N}\left(u_{0}, C(I)\right)+C / N^{3}\right)\right]^{q} \frac{1}{N}\right)^{1 / q} \\
& \quad=\left(\sum_{N=1}^{\infty}\left[N^{\alpha-\frac{1}{q}}\left(C E_{C N}\left(u_{0}, C(I)\right)+C / N^{3}\right)\right]^{q}\right)^{1 / q} \\
& \quad=\left(\sum_{N=1}^{\infty}\left[C N^{\alpha-\frac{1}{q}} E_{C N}\left(u_{0}, C(I)\right)+C / N^{3-\alpha+\frac{1}{q}}\right]^{q}\right)^{1 / q} \\
& \quad=\left(\sum_{N=1}^{\infty}\left[(C N)^{\alpha-\frac{1}{q}} E_{C N}\left(u_{0}, C(I)\right)+C / N^{3-\alpha+\frac{1}{q}}\right]^{q}\right)^{1 / q} .
\end{aligned}
$$

If $0<q<1$, then

$$
\begin{aligned}
{\left[(C N)^{\alpha-\frac{1}{q}} E_{C N}\left(u_{0}, C(I)\right)+C / N^{3-\alpha+\frac{1}{q}}\right]^{q} \leqslant } & {\left[(C N)^{\alpha-\frac{1}{q}} E_{C N}\left(u_{0}, C(I)\right)\right]^{q} } \\
& +\left[C / N^{3-\alpha+\frac{1}{q}}\right]^{q}
\end{aligned}
$$

Therefore,

$$
\begin{aligned}
(5.1 .1) & \leqslant\left(\sum_{N=1}^{\infty}\left[(C N)^{\alpha-\frac{1}{q}} E_{C N}\left(u_{0}, C(I)\right)\right]^{q}+\sum_{N=1}^{\infty}\left[C / N^{3-\alpha+\frac{1}{q}}\right]^{q}\right)^{1 / q} \\
& =\left(\sum_{N=1}^{\infty}\left[(C N)^{\alpha} E_{C N}\left(u_{0}, C(I)\right)\right]^{q} \frac{1}{C N}+\sum_{N=1}^{\infty}\left[C / N^{3-\alpha+\frac{1}{q}}\right]^{q}\right)^{1 / q}
\end{aligned}
$$

Since $u_{0} \in A_{q}^{\alpha}(C(I))$ and $\left\{1 / N^{3-\alpha+\frac{1}{q}}\right\}_{N \geqslant 1} \in l^{q}$ for $0<\alpha<3$,

$$
\begin{gathered}
(5.1 .2) \leqslant 2^{\frac{1}{q}-1}\left[\left(\sum_{N=1}^{\infty}\left[(C N)^{\alpha} E_{C N}\left(u_{0}, C(I)\right)\right]^{q} \frac{1}{C_{2} N}\right)^{1 / q}\right. \\
\left.+\left(\sum_{N=1}^{\infty}\left[C / N^{3-\alpha+\frac{1}{q}}\right]^{q}\right)^{1 / q}\right]<\infty
\end{gathered}
$$


If $1 \leqslant 1<\infty$, then, because $u_{0} \in A_{q}^{\alpha}(C(I))$ and $\left\{1 / N^{3-\alpha+\frac{1}{q}}\right\}_{N \geqslant 1} \in l^{q}$ for $0<\alpha<3$ and by the Minkowski inequality,

$$
\begin{aligned}
(5.1 .1) & \leqslant\left(\sum_{N=1}^{\infty}\left[(C N)^{\alpha-\frac{1}{q}} E_{C N}\left(u_{0}, C(I)\right)\right]^{q}\right)^{1 / q}+\left(\sum_{N=1}^{\infty}\left[C / N^{3-\alpha+\frac{1}{q}}\right]^{q}\right)^{1 / q} \\
& =\left(\sum_{N=1}^{\infty}\left[(C N)^{\alpha} E_{C N}\left(u_{0}, C(I)\right)\right]^{q} \frac{1}{C N}\right)^{1 / q}+\left(\sum_{N=1}^{\infty}\left[C / N^{3-\alpha+\frac{1}{q}}\right]^{q}\right)^{1 / q}<\infty .
\end{aligned}
$$

If $q=\infty$ and $0<\alpha<3$, then

$$
\begin{aligned}
\sup _{N}\left[N^{\alpha} E_{N}\left(u(\cdot, t), C\left(I_{t}\right)\right)\right] \\
\quad \leqslant \sup _{N}\left[N^{\alpha}\left(C E_{C N}\left(u_{0}, C(I)\right)+C / N^{3}\right)\right] \\
\quad \leqslant \sup _{N}\left[N^{\alpha} E_{C N}\left(u_{0}, C(I)\right)\right]+\sup _{N}\left[C / N^{3-\alpha}\right]<\infty .
\end{aligned}
$$

This shows that $u(\cdot, t) \in A P q^{\alpha}\left(C\left(I_{t}\right)\right)$ for $0<\alpha<3$.

Corollary 5.1 follows immediately from Theorem 5.1 and the characterisation of the spaces $A_{q}^{\alpha}\left(C\left(I_{t}\right)\right)$ in terms of Besov-type spaces.

\section{REFERENCES}

[1] C. de Boor, 'Good approximation by splines with variable knots', in Spline Functions and Approximation Theory, (A. Meir and A. Sharma, Editors) (ISNM Birkhäuser-Verlag, Basel, 1973), pp. 57-72.

[2] M.G. Crandall, L.C. Evans and P.L. Lions, 'Some properties of viscosity solutions of Hamilton-Jacobi equations', Trans. Amer. Math. Soc. 282 (1984), 487-502.

[3] M.G. Crandall and P.L. Lions, 'Viscosity solutions of Hamilton-Jacobi equations', Trans. Amer. Math. Soc. 277 (1983), 1-42.

[4] M.G. Crandall and P.L. Lions, 'Two approximations of solutions of Hamilton-Jacobi equations', Math. Comp. 43 (1984), 1-19.

[5] R.A. DeVore, 'Monotone approximation by splines', SIAM J. Math. Anal. 8 (1977), 891-905.

[6] R.A. DeVore and B.J. Lucier, 'High order regularity for conservation laws', Indiana Univ. Math. J. 39 (1990), 413-430.

[7] R.A. DeVore and V.A. Popov, 'Interpolation spaces and non-linear approximation', in Function Spaces and Applications, (M. Cwikel, J. Peetre, Y. Sagher and H. Wallin, Editors), Springer Lecture Notes in Mathematics 1302 (Springer-Verlag, Berlin, Heidelberg, New York, 1988), pp. 191-205. 
[8] R. Jensen and P.E. Souganidis, 'A regularity result for viscosity solutions of Hamilton-Jacobi equations in one space dimension', Trans. Amer. Math. Soc. 301 (1987), 137-147.

[9] S.N. Kružkhov, 'First order quasilinear equations with several independent variables', Math. USSR-Sb. 10 (1970), 217-243.

[10] P.D. Lax, Hyperbolic systems of conservation laws and mathematical theory of shock waves, Regional Conference Series in Applied Mathematics (SIAM, Philadelphia, 1973).

[11] P.L. Lions, Generalized solutions of Hamilton-Jacobi equations, Pitman Research Notes Series (Pitman, London, 1982).

[12] B.J. Lucier, 'A moving mesh numerical method for hyperbolic conservation laws', Math. Comp. 46 (1986), 59-69.

[13] P.P. Petrushev, 'Direct and converse theorems for spline and rational approximation and Besov spaces', in Functions Spaces and Applications, (M. Cwikel, J. Peetre, Y. Sagher and H. Wallin, Editors), Springer Lecture Notes in Mathematics 1302 (Springer-Verlag, Berlin, Heidelberg, New York, 1988), pp. 363-377.

[14] P.E. Souganidis, 'Approximation schemes for viscosity solutions of Hamilton-Jacobi equations', J. Differential Equations 59 (1985), 1-43.

[15] P.E. Souganidis, 'Existence of viscosity solutions of Hamilton-Jacobi equations', J. Differential Equations 56 (1985), 345-390.

Department of Mathematics

Hoseo University

Choongnam 337-850

Korea 